\title{
Theory of conservative diffusion in classical and quantum systems
}

\author{
Zahid Zakir ${ }^{1}$
}

\begin{abstract}
In previous paper [1] conservative diffusion of light particles in a dilute medium of heavy particles was studied by analogue with Brownian motion. In the present paper the theory is formulated in more consistent "hydrodynamic" form by using a conservativity condition only, that mean energy of a light particle is conserved. As a model is taken diffusion of very cold light gas in warm heavy gas a time interval before relaxation when light gas remains cold. Unlike Lorentz's gas, where thermal energies of light and heavy atoms are equal, here their thermal speeds are equal and this leads to the effects of conservativity similar to quantum effects. Such conservative diffusion is described by two equations - the continuity equation and the energy conservation condition, nonlinear under the probability density. At introduction of a complex probability amplitude the equations linearized and turn to the Schrödinger equation. As a result, one must add not probability of alternatives, but probability amplitudes. A free pass length and corresponding momentum define an elementary phase volume and the diffusion coefficient. The predicting new quasi-quantum effects in classical systems are discussed. It is shown that the formalism of quantum mechanics describes the classical conservative diffusion with a constant diffusion coefficient and that quantum mechanics is a particular case of such diffusion in the vacuum where the elementary phase volume of free passage is equal to the Planck constant.
\end{abstract}

PACS: 02.50.Ey, 03.65.Ta, 05.40.Jc

Key words: quantum mechanics, diffusion, Brownian motion, kinetic theory of gases

\section{Content}

Introduction

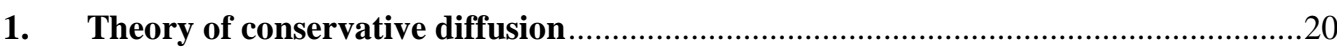

1.1. Microscopic mechanism of conservative diffusion ...........................................20

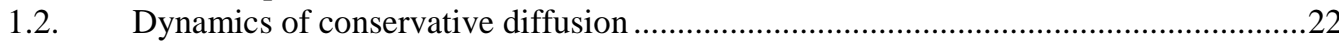

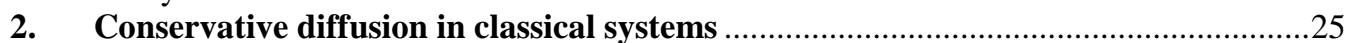

2.1. Diffusion of cold light gas in hot heavy gas before relaxation ................................25

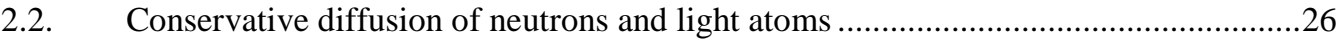

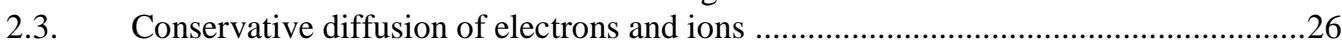

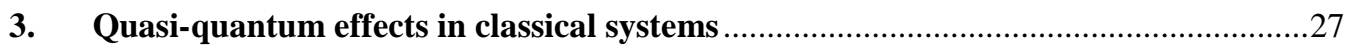

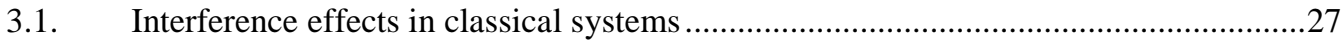

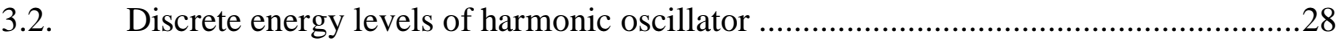

3.3. Discrete energy levels and discrete angular momenta of classical systems ................28

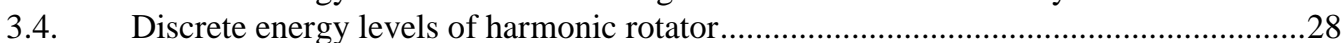

3.5. Quantum statistics in classical systems ...........................................................29

4. The diffusion treatment of quantum mechanics and gravitation ...............................30

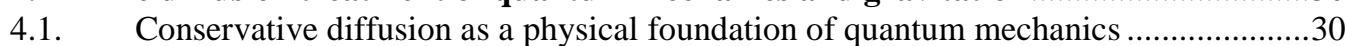

4.2. Gravitation as inhomogeneity of quantum diffusion in vacuum .................................31

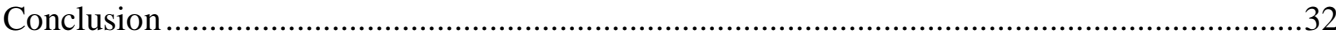

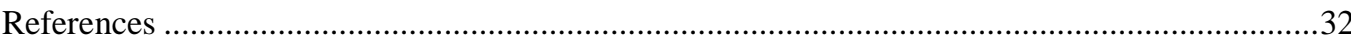

${ }^{1}$ Centre for Theoretical Physics and Astrophyics, Tashkent, Uzbekistan; zahidzakir@ theor-phys.org 


\section{Introduction}

In the previous paper [1] a diffusion in classical systems with low dissipation of particle's energy has been considered and it has been shown that a mechanism of such almost conservative diffusion essentially differs on the mechanism of dissipative diffusion and, at first approximation when dissipation is neglected, there appear qualitatively new effects. However, in the paper it has been used analogue with the Brownian motion, where the Markovian condition for transition probabilities have been postulated [2,3], while in fact at conservative diffusion, as well as in quantum mechanics, they hold only for probability amplitudes [4].

In the present paper, which is radically revised version of [1], the theory of conservative diffusion is formulated by using only a conservativity condition in a "hydrodynamic" form [5] without referring to the stochastic mechanics formalism. Main results of [1] have related by the new physical mechanism, so they have kept the force and justified more consequtively only.

In the theory of random processes and in the theory of condensed states up to now the dissipative diffusion has been studied, mechanism of which is based on the model of a relatively large massive particle randomly walking in a medium of small and light particles [6], when the role of a dissipation of particle's energy is sufficient.

As it has appeared, a microscopic mechanism of conservative diffusion is based on the model of a random walk of a small light particle in dilute medium of massive particles. On a path of free passage a trajectory of the light particle is smooth and at any case it collides only with a single heavy particle of the medium. Assuming the collisions as elastic and recoil as very small, mean energy of the light particle can be taken as conserved at large number of collisions and in this period the diffusion is practically conservative.

The existence of the free passage path, where trajectory is smooth, and also statistical reversibility of the process due to conservation of mean energy lead to two equations - the continuity equation and the energy conservation condition, into which a probability density $\rho(x, t)$ enters non-linearly. At a homogeneous diffusion constant $D=$ const. to joining of two real functions, $\rho$ and an action function for the particle $S(x, t)$, to one a complex amplitude of probability $\psi=\rho^{1 / 2} \cdot \exp (S / 2 m D)$ linearizes these equations and transform them into the Schrödinger equation. As a result, in the conservative classical diffusion one has the probability amplitudes addition law as in quantum mechanics.

In such diffusion the free passage length and a mean square momentum, obeying uncertainty relations, define an elementary phase volume and the diffusion constant. The theory predicts a set of new quasi-quantum effects in classical systems. The conclusions of fundamental character are that formalism of quantum mechanics describes the classical conservative diffusion and that quantum mechanics is only a particular case when the elementary phase volume of free passage is equal to the Planck constant. The theory also allows to treat gravitation as manifestation of inhomogeneity of quantum diffusion in the vacuum [7].

Main relations of the theory are derived in Part 1. In Parts 2 and 3 examples of conservative diffusion are presented and analogues of quantum effects in them (quasiquantum effects) are discussed. In Part 4 the diffusional treatment of quantum mechanics and gravitation are shortly discussed. 


\section{Theory of conservative diffusion}

\subsection{Microscopic mechanism of conservative diffusion}

There are two extreme cases in which diffusion processes qualitatively differ depending on a mass ratio of diffusing particles and particles of the medium.

A microscopic mechanism of dissipative diffusion is based on the model of a large and heavy particle moving in a medium of small and light particles. An elementary realization of such model is diffusion of ideal gas of heavy atoms in gas of light atoms [1]. There it is supposed that a diffusing particle: (1) is larger and heavier than particles of the medium; (2) in any small time interval collides with large number of particles of the medium. At such relation of sizes and masses of particles of the medium and the diffusing particle, very high number of collisions in any time interval, the dissipation of energy of the particle is large and inevitable.

A microscopic mechanism of conservative diffusion is based on an opposite extreme case of diffusion of light particles of small size in a dilute medium of heavy particles. Further considering only such classical systems, we will assume that:

a) a free pass length of particles in medium is larger than size of particles;

b) diffusing particle collides elastically with separate particles of medium;

c) light particle's mass is very small with respect to heavy one: $m \ll M$.

From these properties of such systems follows that:

a) between collisions particles move along smooth classical paths;

b) loss of light particle's energy at such collisions is proportional $m / M$ and insufficient: $\delta E \sim(m / M) \ll E$, and thus in the first approximation we can neglect by dissipation in large enough number of collisions $N \sim(M / m) \gg 1$;

c) spatial size $L_{N D}$ and time interval $t_{N D}$ of a conservativity region, where mean energy is approximately conserved, is inversely proportional to the mass ratio $L_{N D} / l_{D} \sim M / m$, where $l_{D}$ is the free passage length.

At collisions with heavy particles of medium in a rest frame of each molecule mainly changes a direction of velocity of the light particle and a value of its velocity changes insufficiently. But in a laboratory frame, where the box with gas is rested, the fluctuations of light particle's speed is of order of thermal speed of particles of medium and consequently:

$$
\delta v_{D}=V \sqrt{\frac{M}{m}}=\sqrt{\frac{6 k T}{m}},
$$

where $T$ is medium's temperature and $V$ is a thermal speed of the medium's particles.

The external field's contribution can be taken into account easily since all trajectory of a particle consists of stick together smooth paths of free passage between collisions. This is the first basic difference of the mechanism of conservative diffusion from the mechanism of usual diffusion where such paths are absent and one must to smooth them artificially.

Under these requirements the initial energy of light atoms is conserved throughout large enough number of collisions $n_{D} \sim M / m$. In lack of external forces this part of particle's trajectory is $n_{D} l_{D}$ and corresponding time interval $t_{D}$ we will call as conservativity time. 
An effective mean energy of the diffusing particle as sum of thermal energy and mean energy of the external field gained between collisions is conserved and the process is statistically reversible, and there is a symmetry under the time reversal.

Let a light particle starts to diffuse in a dilute medium of heavier particles. By averaging on ensemble of light particles in each moment $t$ a mean length $l_{D}$, a mean time $\tau_{D}$ and a mean velocity $v_{D}=l_{D} / \tau_{D}$ of free passage can be defined. The last one is related to a mean momentum and particle's kinetic energy as usual $p_{D}=m v_{D}, E_{D}=m v_{D}^{2} / 2$.

During the conservativity time the ensemble of light particles is far from thermodynamic equilibrium with medium. Opposite to the Lorentz gas, where thermal energies of light and heavy particles are equal, in our case only their thermal velocities are equal. For this reason the thermal energy of light particles, as well as temperature of the light gas, remain sufficiently lower than for the heavy gas.

The existence of classical parts of a trajectory with the singled out mean characteristics $l_{D}, p_{D}$ is a characteristic property of conservative diffusion, while for usual diffusion they were unnecessary. In particular, due to conservation of particle's mean energy, it is possible to introduce mean value of an abbreviated action function $S$ in the time interval $t^{\prime}-t=N \tau_{D}, N \gg 1$. It represents the sum over short classical parts of the trajectory:

$$
\Delta S=N S_{D}, \quad S_{D}=p_{D} l_{D},
$$

where $S_{D}$ is an elementary abbreviated action of the medium separating a region of classicality of trajectories from a region of stochasticity.

However, statistical mechanics deals with not by the action function along trajectories, but with a value of a phase volume $\Delta \Gamma=\Delta p \Delta x$ in which a particle is placed during a time slice $\Delta t$. In our case the particle at free passage time $\tau_{D}$ places in a sell of size equal to the free pass length $l_{D}$ and has the mean momentum $p_{D}=m l_{D} / \tau_{D}$. Then for such particle there is an elementary phase volume:

$$
\Delta \Gamma=p_{D} l_{D}=\frac{m l_{D}^{2}}{\tau_{D}}=\Gamma_{D},
$$

which coincides with the elementary abbreviated action $S_{D}$ and also separates the classicality region of trajectories on the stochasticity region.

Since $\Gamma_{D}$ plays such essential role, be as the elementary phase volume for the particles, it will be more natural to take it as primary quantity and to express through it other kinematical and dynamical characteristics of the system. In particular, from (3) and the definition of the diffusion constant $l_{D}^{2}=2 v \tau_{D}$ follows

$$
l_{D}^{2}=\frac{\Gamma_{D}}{m} \tau_{D}=2 v \tau_{D}, \quad v=\frac{\Gamma_{D}}{2 m},
$$

i.e. the diffusion constant $2 v$ in our system is in fact elementary phase volume $\Gamma_{D}$ for a unit mass particle. 
Zakir Z. (2014) Theoretical Physics, Astrophysics and Cosmology, 9, 1, TPAC: 4874-036

\subsection{Dynamics of conservative diffusion}

In theories of Brownian motion (dissipative diffusion) for determination of speeds and dispersions the realvalued transition probabilities for probability density have been introduced. The former formulation - stochastic mechanics - also has been based on introduction of the same transition probabilities by postulating the Markovian property by direct analogy to the Brownian motion [2]. But then, because of statistical reversibility in time of conservative diffusion, it was necessary to operate in the equations at the same time with probabilities of transition forward and back in time [3]. It has led to a non-Markovian process because of (indirect) dependence of an increment $x(t+\Delta t)-x(t)$ on the former increment $x(t)-x(t-\Delta t)$ and this means an intrinsic contradiction in the theory [4].

In the present paper the theory of conservative diffusion is formulated not by analogy to the formalism of Brownian motion, but on the basis of the hydrodynamic analogy [5] which is more natural for conservative diffusion because of existence of the free passing paths.

Speed of drift $\mathbf{v}(x, t)$ is that part of speed in the ensemble of particles which at large mass passes to usual classical speed. Since particle's trajectory between collisions is classical, therefore, as well as in the Hamiltonian dynamics, the drift component of the momentum $\mathbf{p}_{\mathbf{v}}=m \mathbf{v}$ can be represented as a gradient of some function of coordinates and time $S(x, t)$ :

$$
\mathbf{p}_{\mathbf{v}}=m \mathbf{v}(x, t)=\nabla S(x, t) .
$$

Mean speed of diffusion $\mathbf{u}(x, t)$ of the particle is that part of the speed which caused by fluctuations and for this reason by definition its average over ensemble vanishes, while rootmean-square value is non-zero:

$$
\overline{\mathbf{u}}=\int \mathbf{u} \rho d^{3} x=0, \quad \int \mathbf{u}^{2} \rho d^{3} x \neq 0 .
$$

Here the probability density $\rho(x, t)$ is normalized as:

$$
\int \rho(\mathbf{x}(t), t) d x(t)=1,
$$

and, due to conservation of probability, satisfies the continuity equation:

$$
\frac{\partial \rho}{\partial t}+\nabla \cdot(\mathbf{v} \rho)=0
$$

Vanishing of the mean value $\overline{\mathbf{u}}$ is provided by vanishing of the volume integral (6) of the function $\mathbf{u} \rho$. It testifies that $\mathbf{u} \rho$ represents a gradient of a certain function $\nabla f(x, t)$, and also that function $f$ should disappear at spatial infinity, providing vanishing a surface integral at boundary $\Sigma$ of the volume integration. It means that $f$ is proportional to $\rho$ which under the normalization condition (7) just disappears at spatial infinity. By denoting the rest of $f$ as $-D(x, t)$, where minus sign is chosen for coinciding by an ordinary diffusion flux directed backward to the gradient of concentration, we have $f(x, t)=-\rho(x, t) D(x, t)$. Thus, a dependence between $\mathbf{u}$ and $\rho$ follows from the general properties of these functions and is determined up to a certain function $D(x, t)$ which will appear further as the diffusion coefficient. Thus, from: 


$$
\overline{\mathbf{u}}=\int \mathbf{u} \rho d^{3} x=-\int \frac{\nabla(\rho D)}{\rho} \rho d^{3} x=-\int_{\Sigma} \rho D d \Sigma=0 .
$$

we obtain an expression connecting $\mathbf{u}$ with $\rho$ :

$$
\mathbf{u}(x, t)=-\frac{\nabla(\rho D)}{\rho}, \quad \mathbf{p}_{u}=m \mathbf{u},
$$

where $\mathbf{p}_{u}$ is diffusion component of momentum obeying the uncertainty relation (at $\overline{\mathbf{x}}=0$ )

$$
\sqrt{\overline{\mathbf{p}_{u}^{2}} \cdot \overline{\mathbf{x}^{2}}} \geq\left|\overline{\mathbf{p}_{u} \cdot \mathbf{x}}\right|=m\left|\int \mathbf{u} \cdot \mathbf{x} \rho d^{3} x\right|=m\left|\int(\nabla(\rho D)) \cdot \mathbf{x} d^{3} x\right|=m \bar{D} .
$$

Taking into account that kinetic energy of a particle is a sum of drift and fluctuating parts, for the Hamiltonian we have the expression:

$$
H=\int\left(\frac{\mathbf{p}_{v}^{2}}{2 m}+\frac{\mathbf{p}_{u}^{2}}{2 m}+V\right) \rho d^{3} x
$$

which at $D=$ const. and using (5) and (10) can be written in terms $S$ and $\rho$ as:

$$
H=\int\left(\frac{1}{2 m}(\nabla S)^{2}+\frac{m D^{2}}{2}\left(\frac{\nabla \rho}{\rho}\right)^{2}+V\right) \rho d^{3} x,
$$

In this "hydrodynamic" Hamiltonian $H$ two functions, $S(x, t)$ and $\rho(x, t)$, play the role of canonical variables. Defining Poisson's brackets in terms of this "hydrodynamic" canonical pair [8] as:

$$
\begin{gathered}
\{A, B\}=\int\left(\frac{\delta A}{\delta \rho} \frac{\delta B}{\delta S}-\frac{\delta B}{\delta \rho} \frac{\delta A}{\delta S}\right) d^{3} x, \\
\left\{\rho(x), S\left(x^{\prime}\right)\right\}=\delta\left(x-x^{\prime}\right),
\end{gathered}
$$

we obtain corresponding canonical equations:

$$
\begin{gathered}
\frac{\partial S}{\partial t}=\{S, H\}=-\frac{\delta H}{\delta \rho}, \\
\frac{\partial \rho}{\partial t}=\{\rho, H\}=\frac{\delta H}{\delta S} .
\end{gathered}
$$

By inserting the expression for $H$ from Eq. (13), we see that these canonical equations turn to a well-known "hydrodynamic" representation of the Schrödinger equation [5]:

$$
\begin{gathered}
\frac{\partial S}{\partial t}+\left(\frac{(\nabla S)^{2}}{2 m}+V\right)-\frac{(2 m v)^{2}}{2 m} \frac{\Delta \sqrt{\rho}}{\sqrt{\rho}}=0, \\
\frac{\partial \rho}{\partial t}+\frac{1}{m} \nabla \cdot(\rho \nabla S)=0 .
\end{gathered}
$$

The first equation differs from the Hamilton-Jacobi equation only by the last non-linear term non-linear under the probability density $\rho$. As the result, at conservative diffusion probabilities of two alternatives does not obey the classical probability addition law:

$$
\rho \neq \rho_{1}+\rho_{2}
$$

But the system of equations (18)-(19) admits a linearization by a canonical transformation from the pair of real-valued functions $\rho(x, t), S(x, t)$ to one a complex function $\psi(x, t)[3,8]$ : 
Zakir Z. (2014) Theoretical Physics, Astrophysics and Cosmology, 9, 1, TPAC: 4874-036

$$
\begin{gathered}
\psi=\sqrt{\rho} \exp (i S / 2 m D), \quad \psi^{*}=\sqrt{\rho} \exp (-i S / 2 m D), \\
\rho=\psi^{*} \psi, \quad S=i(2 m D) \cdot \ln \left(\psi^{*} / \psi\right) .
\end{gathered}
$$

The Poisson brackets then is defined as:

$$
\begin{gathered}
\{A, B\}=(i \cdot 2 m D)^{-1} \int\left(\frac{\delta A}{\delta \psi} \frac{\delta B}{\delta \psi^{*}}-\frac{\delta B}{\delta \psi} \frac{\delta A}{\delta \psi^{*}}\right) d^{3} x, \\
\left\{\psi^{*}(x), \psi\left(x^{\prime}\right)\right\}=\delta\left(x-x^{\prime}\right) / i(2 m D),
\end{gathered}
$$

and the Hamiltonian (13) takes the form

$$
H=\int \psi^{*} \hat{H} \psi d^{3} x, \quad \hat{H}=-\frac{(2 m D)^{2}}{2 m} \Delta+V .
$$

The canonical equations (16)-(17) transform to:

$$
\begin{gathered}
\frac{\partial \psi}{\partial t}=\{\psi, H\}=\frac{1}{i(2 m D)} \frac{\delta H}{\delta \psi^{*}}, \\
\frac{\partial \psi^{*}}{\partial t}=\left\{\psi^{*}, H\right\}=-\frac{1}{i(2 m D)} \frac{\delta H}{\delta \psi} .
\end{gathered}
$$

These equations, after substituting of the expression for $H(25)$, take the form of the Schrödinger equation for the "wave function" $\psi(x, t)$ :

$$
i(2 m D) \frac{\partial \psi}{\partial t}=\hat{H} \psi
$$

A physical meaning of wave behavior consists in periodic repetition along a trajectory of parts of free passage by mean length $l_{D}$, and also in the existence of an elementary phase space volume $\Gamma_{D}$ related by this free pass length.

Unlike $\rho$, the amplitude of probability density $\psi(x, t)$ obeys the linear equation and it takes place the superposition of states:

$$
\psi=c_{1} \psi_{1}+c_{2} \psi_{2} \text {. }
$$

As the result, at conservative diffusion one should add not probabilities of two alternatives, but their probability amplitudes.

The solution of Eq. (28) in the integral form looks like:

$$
\psi(x, t)=\int G\left(x, t ; x_{0}, t_{0}\right) \psi\left(x_{0}, t_{0}\right) d^{3} x_{0},
$$

where the complex amplitude of transition probabilities $G\left(x, t ; x_{0}, t_{0}\right)$ satisfies to the equation:

$$
G\left(x, t ; x_{0}, t_{0}\right)=\int G\left(x, t ; x^{\prime}, t^{\prime}\right) G\left(x^{\prime}, t^{\prime} ; x_{0}, t_{0}\right) d^{3} x^{\prime}, \quad t>t^{\prime}>t_{0} .
$$

Thus, at conservative diffusion the Markov condition satisfy only amplitudes of transition probabilities, which are complex valued.

Thus, the conservative diffusion in classical systems at the conditions mentioned in the beginning of the section is described by the formalism of quantum mechanics with replacement $\hbar \rightarrow 2 m D$. And vice verse, quantum mechanics appears as a special case of conservative diffusion of classical particles in the physical vacuum with the fixed diffusion coefficient $D=\hbar / 2 m$. 


\section{Conservative diffusion in classical systems}

\subsection{Diffusion of cold light gas in hot heavy gas before relaxation}

The classical model of ideal gas is based on the assumptions that between collisions atoms move along classical trajectories, collisions of atoms are elastic and their mean kinetic energy is conserved.

For realisation of the conservative diffusion it is suitable a mixture of two ideal oneatomic gases when masses of two kind atoms sufficiently differ and concentration of the light component is small. At diffusion of light gas in heavy we assume that to above mentioned conditions of ideality of gases additional requirement are added, that the light atoms of mass $m$ collide mainly with heavy atoms of mass $M$ and at $m / M \ll 1$ when recoil is very small.

Kinetic theory of dissipative diffusion of light gas in the heavy, developed by Lorentz [6], concerns to a mixture in thermodynamic equilibrium when thermal energies of both components are equal. In this case the thermal velocity of light atoms is so large that the heavy atoms can be taken as rested. This very simplifies calculation of transport coefficients, including the diffusion coefficient.

Diffusion of a cold light gas in a hot heavy gas is conservative in that small, but finite time interval up to relaxation when both components are still far from thermodynamic equilibrium. Below we will consider essentially new effects of conservativity only .

In ordinary gases with heavy molecules at diffusion of atoms of light elements a conservativity region is of order $L_{N D} \sim\left(10^{2} \div 10^{3}\right) l_{D}$ and $t_{N D} \sim L_{N D} / \bar{v}$, where $\bar{v}$ - mean square velocity of a light atom. These sizes and times are small for gases in a normal state, but for dilute gases the sizes are comparable to sizes of a container and times are sufficient for passing by the light atoms the same distance.

At the conservative diffusion region, before thermodynamic equilibrium, a mean square value of velocity of light atoms is the same as a mean squire velocity of heavy gas molecules and, thus, the fluctuation of energy of the light atoms is of order:

$$
\delta E \simeq \frac{1}{2} m \overline{V^{2}}=\frac{m}{M} \frac{1}{2} M \overline{V^{2}}=\frac{m}{M} \frac{3}{2} k T .
$$

The existence of a gradient of concentration of light gas $\nabla n$ generates the diffusion flux $n \mathbf{u}$ smoothing concentration and directed in the opposite direction:

$$
n \mathbf{u}=-D \nabla n .
$$

As easy atoms are considered facing only with heavy, each easy atom makes casual wanderings irrespective of the others. Therefore, as well as in case of a Brown particle, instead of concentration $n(x, t)$ it is possible to operate with probability density $\rho(x, t)$ for one particle. Then instead of a diffusion flux of many particles we will deal with a probability flux for one particle:

$$
\rho \mathbf{u}=-D \nabla \rho,
$$

from which it follows the speed of diffusion $\mathbf{u}$ from Eq. (10) (at $D=$ const.).

The non-trivial mechanism of conservative diffusion allows one to reveal a number of new effects which can be observed under the corresponding conditions, particularly:

1) mean energy and mean velocity of drift of light atoms and also their drift acceleration are defined mainly by an external field, the thin flat layer of light gas can enter in a volume with 
Zakir Z. (2014) Theoretical Physics, Astrophysics and Cosmology, 9, 1, TPAC: 4874-036

heavy gas from one partition wall and escape it (with particular losses) from other partition wall by means of the diffusion mechanism (without formation of flows in the medium);

2 ) in the conservativity region the light gas is not in thermodynamic equilibrium with heavy gas, thus a dependence of a diffusion flux on temperature of the medium is sufficiently weaker, than at usual equilibrium requirements;

3) ordinary properties of a diffusion flux of a light gas in heavy are related mainly with a vacuity of a low temperature medium (low pressure or low density) effects, while the conservativity effects can be realized even at higher temperatures and densities of the medium;

4) some known phenomena, specific to gases at very low pressures, can be realized at more high pressures also with a pressure "growing" coefficient of order $\sim M / m$;

5 ) there should be added the probability amplitudes and in concentration distribution and diffusion flux of light atoms the wave properties (quasi-quantum effects) will be exhibited determined by the diffusion constant.

\subsection{Conservative diffusion of neutrons and light atoms}

Requirements for realization of conservative diffusion of neutrons in condensed media of heavy nuclei are, on the one hand, more favorable, than for diffusion of atoms, since the scattering happens on the nuclei. Because of small cross-section of nuclei and neutrons the free length should be large only in comparison with sizes of nuclei that is satisfied almost always, except neutron stars only.

On the other hand, for particular matter with heavy enough nuclei it is necessary to find a region of energies of diffusing neutrons when ionization loss is insufficient and a quantummechanical wavelength is small enough, so that neutrons can be considered as classical particles. Number of collisions $N_{(1)} \sim M_{(1)} / m_{n} \sim 10^{2} \div 10^{3}$ defines a region conservativity when recoil at neutron's scattering on a nucleus is transferred only to the nucleus.

If recoil is transferred to a molecule or a cluster with $k$ nuclei with total mass $M_{(k)}$, a number of collisions in the region conservativity $N_{(k)} \sim M_{(k)} / m_{n} \sim 10^{3} \div 10^{2 k}$ can be large.

At last, when recoil at neutron's scattering is transferred to whole crystal of total mass $M$, a region of conservativity practically will coincide with crystal sizes and it is possible to consider the collisions of neutrons as elastic with very high accuracy. This is an effect inverse to the Mössbauer effect.

Thus, the new effects of conservative diffusion of baryons and light nuclei are:

(1) capabilities to drive flows of neutral particles by regulating a composition, density and temperature of the medium;

(2) occurrence of an effect inverse to the Mössbauer effect with a macroscopic region of conservativity;

(3) in the medium from heavy elements it is possible to study possibilities of delivery of baryons and impurities of light elements into a definite region with a given distribution.

\subsection{Conservative diffusion of electrons and ions}

In a weak ionized gas concentrations of electrons and ions are small and a number of collisions of electrons with electrons and ions is much less than number of collisions of electrons with neutral molecules. For low energy electrons, when they do not excite or ionize 
the molecules, the collisions of electrons with molecules are elastic [6] and due to small mass ratio of electron and molecule, at collisions electron's energy practically does not change.

For this reason at conservative diffusion motion of electrons in external electrical and magnetic fields is described by equation of motion with a mean drift acceleration $\mathbf{a}$ equal to:

$$
m \mathbf{a}=e \mathbf{E}+\frac{e}{c}[\mathbf{v H}] .
$$

As a result, effects conservativity will be exhibited in the form, that:

(1) flow of accelerated electrons in the electric field and a current strength increase on an exit because of higher drift velocity of electrons;

(2) in magnetic field the effects leading to increasing circular speed will be exhibited;

(3) a conservative conductivity in various condensed media which is close to hightemperature superconductivity, since here former limitations will be partially removed and essentially new effects can be revealed;

(4) new effects in structures of micro- and nano-ecectronics.

\section{Quasi-quantum effects in classical systems}

\subsection{Interference effects in classical systems}

At conservative diffusion the probability amplitudes addition law holds which firstly have been discovered in quantum systems and taken as one of founding principles of their theory, as the principle of superposition. As it will becomes clear, this property is characteristic for all systems with conservative diffusion.

For this reason, in the classical systems, considered in the first section, consequences of the probability amplitudes addition law, in particular, interference effects for a probability distribution, will appear also.

At diffusion of light classical particles in a medium of heavy ones in a rectangular box we can consider ensemble of light particles which having started to diffuse with the left wall of the volume with constant drift speed $v_{x}$ along $x$ axis, at given time have reached the right wall.

Further we will consider a standard two slit interference experiment, but dealing with classically moving particles, quantum mechanical wavelength of which is very small than size of the slits, and instead of the Planck constant there figures a diffusion constant $\hbar \rightarrow 2 m D$. The interference picture will correspond thus to a probability wave with a wavelength:

$$
\lambda=2 \pi \cdot \frac{2 m D}{p_{x}}=2 \pi \frac{\Gamma_{D}}{p_{x}} .
$$

On the first and second series of experiments we will place in the middle of the volume a partition wall with a narrow slit, at first, above the wall's middle and then below the middle. Then in distribution of the passing light atoms, registered on the right wall, there will be maximums opposite to that from two slits which has been open.

At last, on the third series of experiments we will dispose the partition wall with two open slits. Then, if diffusion of light particles in the given medium is adequately close to conservative, the interference picture in the probability distribution of the passing particles registered on the right wall will be observed.

The wave behavior of ensemble of classically moving light particles on this experiment obviously demonstrates qualitative difference of conservative diffusion of light particles in medium from heavy ones from usual dissipative diffusion of heavy particles in a medium of light ones. Notice, that as ensemble of light particles there must be understand multiple repeated 
Zakir Z. (2014) Theoretical Physics, Astrophysics and Cosmology, 9, 1, TPAC: 4874-036

diffusion of one kind of particles in the given region with heavy gas, and here the interference picture is the result of addition in this ensemble of probability amplitudes of two alternatives of transition through two slits.

\subsection{Discrete energy levels of harmonic oscillator}

Let us consider in the same volume conservative diffusion of a light charged particle at the presence of an external electromagnetic field, so that the particle make transverse harmonic oscillations around a central horizontal axis of the box. Since this diffusion process is described by the Schrödinger equation, the energy levels of such oscillations will be discrete:

$$
E_{n}=(2 m D) \omega(n+1 / 2) .
$$

It will be possible to measure experimentally the discrete mean energy of particles at vertical oscillations in addition to continuous energy of horizontal drift. It can be registered, for example, on the Doppler line broadening of radiation of oscillating light ions. Maximums of vertical deviations will be approximately discrete also.

The presence of the «zero-point energy» of oscillations in this case expresses nonlocalizability of ensemble of diffusing classical particles due to the uncertainty relation (11).

At first sight it seems paradoxical since the trajectories of light particles have classical parts between collisions where their coordinates and momenta can be defined "precisely" (at comparison with a free length and a mean momentum).

However, the ensemble of such particles is characterized by a deviation of trajectories and a probability amplitude with a particular wave vector. The diffusion flux is directed to smoothing of the probability density, i.e. against its gradient $-\nabla \rho$. Therefore, at localization of the ensemble of diffusing particles in a finite region, mean square deviations of coordinates and momenta in the ensemble appear related by the uncertainty relation. Then a localization region of the ensemble small, then it is higher uncertainty of momentum or diffusion flux.

Thus, the measuring of lowest energy level of harmonic oscillations in this experiment confirms the uncertainty relation for conservative diffusion in classical systems.

\subsection{Discrete energy levels and discrete angular momenta of classical systems}

Construction of a macroscopic analogues of quantum systems at diffusion of a light classical particle in an external field where energy levels and an angular momentum are discrete, appears now related only with selection of medium of heavy particles, diffusing light particles and corresponding configuration of external fields.

In particular, the angular momentum discreteness will appear in "filling" of levels of hydrogen-like "quasiatom" at dissipation of "test" diffusing particles on these structures and also at the presence of magnetic field.

Macroscopic analogues of hydrogen-like and more complex "quasiatoms" and also discreteness of levels in magnetic field can appear useful both in technological problems and at visual demonstrating of earlier difficult explainable aspects of quantum mechanics.

\subsection{Discrete energy levels of harmonic rotator}

In the volume of heavy molecules we will consider conservative diffusion of the light charged particles in an external field so that the particle made harmonic rotations around the vertical axis directed along a field. 
Energy of such harmonic rotator [9] is proportional to the angular moment $M_{n}$ :

$$
E_{n}=\left|M_{n}\right| \omega .
$$

Quantization of energy is thus related by quantization of the angular momentum which on the plane of rotation is simply multiple to an integer: $M_{n}=(2 m D) n$., where $n=0, \pm 1, \ldots$

Thus, the energy spectrum of the harmonic rotator is also discrete and it differs on the spectrum of the harmonic rotator by the lack of the zero point energy:

$$
E_{n}=(2 m D) \omega n \text {. }
$$

At real experiment, of course, one must take into account radial oscillatory modes too which contain zero point energy.

In the external magnetic field energy spectrum of such magneto-harmonic rotator has the form [13]:

$$
E_{n}=m D \omega \cdot\left(\left|m_{H}\right|+m_{H}\right) .
$$

\subsection{Quantum statistics in classical systems}

Above we considered an ensemble of light particles, assuming that any light particle collides only with single heavy particle of medium, i.e. it is a one-particle problem. Let us consider multi-particle problems where concentration of light particles is not small, collisions between them are essential and we come to the problem about ideal gas of light particles making conservative diffusion. Since at collisions of light particles with each other their energy redistributes, these light particles come to thermodynamic equilibrium among themselves promptly, than with the medium.

Earlier such problem was considered at electron's diffusion in medium of neutral atoms or ions [6]. There a temperature $T_{e}$ of electronic gas sufficiently differs on medium's temperature $T_{i}$. If earlier in the equilibrium state it was studied the case $T_{e} \gg T_{i}$, for us it is interesting an opposite situation $T_{e} \ll T_{i}$ when the conservative diffusion of electrons is realized, which in fact is also a new mechanism of superconductivity.

For our purposes it is interesting the fact that gas of light particles in medium of the heavy will be described by quantum statistics where the role of the Planck constant plays a diffusion constant $\hbar \rightarrow 2 m v$. Manifestations of effects of quantum statistics will be numerous and for this reason we consider some of them only which demonstrate main properties of quantum statistics.

The first property is indistinguishability. If in multiparticle system energy levels have equal probability and particles are assumed distinguishable, one has the Boltzman distribution:

$$
n=n_{0} e^{-E_{n} / k T} .
$$

If levels have equal probability also, but particles are indistinguishable, the Bose-Einstein distribution will be obtained.

$$
n=\frac{n_{0}}{e^{E_{n} / k T}-1} .
$$

Therefore there is a question how in system of classical distinguishable particles can appear quantum statistics?

The solution to this problem has been discovered by Terzoff and Bayer [10] and consists in that the assumption about equal probability of levels appears too strong limitation. In fact, as Bose did from the beginning, there is enough an assumption that sum of probabilities 
Zakir Z. (2014) Theoretical Physics, Astrophysics and Cosmology, 9, 1, TPAC: 4874-036

of all alternative ways of fillings is equal to unity and that system's total energy, obviously, does not change at all possible fillings:

$$
E=\sum_{n} N_{n} E_{n} .
$$

At such general assumptions, as it has appeared, in gas from distinguishable particles the BoseEinstein distribution generally takes place and only at the additional equal probability requirement it is narrowed down to the Boltzmann distribution [10]. Thus, the indistinguishability of particles in the quantum statistics appears effectively, being a consequence of certain limitations for system from distinguishable particles.

The second property of quantum statistics which we will consider is an analogue of the Bose-Einstein condensation for light gas at conservative diffusion in heavy gas. This effect can be observed by creating conditions similar to usual quantum effect, but with replacement $\hbar \rightarrow 2 m v$. It means that a quasi-Bose-Einstein condensation can be realized on macroscopic systems also, by choosing a content of a medium and a diffusing light gas.

The third in essence new property is the Pauli's exclusion principle for particle systems which are described by wave functions, antisymmetric at transpositions of particles. Still neglecting details of connection of spin and statistics, here we notice only that the theory of conservative diffusion generally admits such states irrespective of the reasons of their origin. Thus, if at conditions where there is diffusion with such wave functions, the consequences of the Fermi-Dirac statistics and Pauli's principle can be observed in classical systems too.

\section{The diffusion treatment of quantum mechanics and gravitation}

\subsection{Conservative diffusion as a physical foundation of quantum mechanics}

Quantum mechanics is a theoretical basis of a modern physics and reliability of its formalism (in various formulations) for description of physical phenomena is beyond doubt.

However concerning a physical interpretation of quantization the situation is opposite and there is common consent that in that aspect quantum mechanics is far of to be finished.

The standard interpretations of quantum mechanics are based on in fact ideological statement that we should divide the reality on macro- and microscopic objects and for the last ones should restrict classical mechanics and to add something new. In this sense they are in fact "revolutionary" approaches. However there are many questions, on which they do not answer, postulating the necessary answer, or giving inconsistent answers:

(1) Why quantization is necessary?

(2) Why there are quantum fluctuations and what they mean?

(3) What in fact fluctuates: a background, acting on a particle, or the particle in itself at a smooth background?

(4) Why there should be added probability amplitudes of alternative events, instead of probabilities?

(5) What principally distinguishes quantum particles from the classical ones?

(6) If there are large fluctuations of energy and momentum of a particle, what is a source of these large energy and momentum?

(7) Is such violation of energy and momentum conservation compensated by decreasing of energy and momentum of something?

(8) Are fluctuations related to structure of spacetime or not? 
Existence or lack of answers to these questions practically does not influences to efficiency of applications of quantum mechanics and thus such searches did not represented a particular interest. However, from the point of view of prospects of further development of quantum theory the correct answers are necessary and important.

The diffusion treatment, in which quantum fluctuations are reduced to a conservative diffusion, in that sense represents a sufficient progress. This version of quantum mechanics is a single one which is free on the "ideological" separating of reality to macro- and micro-objects.

In former attempts in this direction [2,3], from one side a microscopic mechanism of conservative diffusion in classical systems was unclear and from other side the formalism was not fully consistent [4].

Thus, the next step was consisted, starting from a clear physical picture, to construct a microscopic theory of such diffusion which would be consistent and compatible with quantum mechanics, which exactly what is considered above in the present paper. As it appears, at first, the conservative diffusion can be easily realized in classical systems, but under the conditions different from conditions for ordinary Brownian motion and, secondly, quantum-mechanical formalism naturally follows from the theory of diffusion in such systems.

In contrast to previous interpretations, the theory of conservative diffusion is based on a realistic point of view and and naturally answers to most of questions which seemed as mysterious for the former interpretations. In this treatment there are no special quantum objects and it is based on the physical fact that at motion in the physical vacuum classical particles possesses specific fluctuations with magnitude inversely proportional to their mass.

Thus, the vacuum properties are assumed such that at interaction with it:

(1) spatial coordinates of a particle fluctuate, so that particle's motion represents a specific diffusion process;

(2) the equations describing the diffusion of the particle are reversible in time that further leads to conservation of mean energy of the particle;

(3) elementary phase volume, characteristic for the coservative diffusion, is universal and equal to the Planck constant $\hbar$.

In such diffusional treatment the answers to the problems 1-3 are given the same as in any system of interacting particles and fields, i.e. by the fact of the existence of a media with particular properties.

But to problems 4-5, key and most mysterious ones in all previous treatments, an answer is simple and clear. As it has been shown in Part 1, from the non-linear equations of conservative diffusion, relating a probability density and action function of classical particles, there follow the linear Schrödinger equation for a complex probability amplitude.

Answers to other three questions 6-8, requiring consideration of a more general form of diffusion, we will discuss in the next section.

\subsection{Gravitation as inhomogeneity of quantum diffusion in vacuum}

The large fluctuations of energy of a particle happen due to interaction with the physical vacuum and, consequently, at any moment growing of energy of the particle is compensated by corresponding local decreasing of energy of the physical vacuum and vice versa [7]. It is the answer on 6th and 7th problems.

The fact that growing of particle's energy at a quantum fluctuation should lead to decreasing of energy of the physical vacuum to the same value has a direct observable consequence in the form of a diffusional treatment of gravitation. Such treatment answers to 78th problems too. 
Zakir Z. (2014) Theoretical Physics, Astrophysics and Cosmology, 9, 1, TPAC: 4874-036

At large concentration of particles in a small region of space the vacuum energy becomes reduced at any moment and here the mean vacuum energy becomes lower than at distant regions without particles. Due to decreasing of vacuum energy level the intensity of fluctuations of particles is reduced too, which then appears as decreasing of a diffusion constant.

It leads to slowing down of rate of all quantum processes, thus frequencies will be redshifted, proper times will be dilated. As the result, particles from other regions with normal fluctuations begin slowly drift to this region of dilated fluctuations.

All these are the characteristic properties of gravitation which in the diffusional treatment is considered as manifestation of inhomogeneity of conservative diffusion in vacuum. Detail discussion of the diffusion treatment of gravitation is presented in the next paper [7].

\section{Conclusion}

Thus, in low-dissipative systems always there is a region of conservativity where throughout large number of collisions it is possible to neglect by dissipation. A model of such conservative diffusion is diffusion of a light particle in a dilute medium of heavy particles. In the conservativity region of such system a mean energy of the light particle is conserved and the diffusion process is described by more general theory with equations symmetric under both directions of time. Thus, there is the elementary phase volume $\Gamma_{D}=p_{D} l_{D}$ of paths of free passage of the light particle and a diffusion constant then is equal to $v=\Gamma_{D} / 2 m$.

Main difference of the theory of conservative diffusion from the usual consists that in this theory there should be added probability amplitudes instead of probabilities. There holds a superposition principle for these amplitudes since non-linear diffusion equations for forward and backward in time transitions are linearized, turning to the complex Schrödinger equation. There are also the uncertainty relations for uncertainties of coordinates and momenta in the ensemble of particles, restricting product of their variances by the elementary phase volume.

Thus, it has appeared that the formalism of quantum mechanics has wider area of applicability than especially quantum systems and describes in fact a classical conservative diffusion with a homogeneous diffusion constant, realized at diffusion of light particles in dilute medium of heavy particles. Quantum mechanics is only a particular case of such diffusion with the elementary phase volume $\Gamma_{D}=\hbar$.

Examples of classical systems with larger elementary phase volume show that the effects of conservativity are nontrivial, enough universal and represent a new area of research with observable consequences and many possible applications. As most known consequence of the conservative diffusion appears gravitation which in diffusion treatment relates by inhomogeneous diffusion in vacuum [7].

\section{References}

1. Zakir Z. (2009) Theor. Phys., Astrophys. and Cosmol. 4(3) 1, doi: 10.9751/TPAC.2798-011.

2. Fenyes, I. (1952). Z. Phys. , 132, 81.

3. Nelson E. (1966) Phys. Rev., 150, 1057; (1985) Quantum Fluctuations. Pr.U.P.

4. Grabert H., Hänggi P., Talkner P. (1979) Phys. Rev. A19, 2440.

5. Madelung E. (1926) Naturwiss. 14, 1004.

6. Landau L.D., Lifshitz E.M. (1981) Physical Kinetics. Pergamon.

7. Zakir Z. (2014) Theor. Phys., Astrophys. and Cosmol. 9(1) 33, doi: 10.9751/TPAC.4874-037.

8. Guerra F., Marra R. (1983) Phys. Rev. D28, 1916. 
9. Zakir Z. (2011) Theor. Phys., Astrophys. and Cosmol. 6(2) 31, 10.9751/TPAC.3900-022.

10. Tersoff J., Bayer D. (1983) Phys. Rev. Lett. 50, 8, 553. 